\title{
NONLINEAR VIBRATIONAL AND ROTATIONAL ANALYSIS OF MICROBEAMS IN NANOBIOMATERIALS USING GALERKIN DECOMPOSITION AND DIFFERENTIAL TRANSFORM METHODS
}

\author{
Olurotimi Adeleye \\ Department of Biomedical Engineering, University of Lagos \\ Akoka, Lagos, Nigeria \\ oadeleye@unilag.edu.ng \\ Abdulahi Atitebi \\ Department of Systems Engineering, University of Lagos \\ Akoka, Lagos, Nigeria \\ ibnaganiyy@gmail.com \\ Ahmed Yinusa \\ Department of Mechanical Engineering, University of Lagos \\ Akoka, Lagos, Nigeria \\ aayinusa@unilag.edu.ng
}

[Received: September 1, 2020; Accepted: March 15, 2021]

\begin{abstract}
In this paper, a nonlinear vibrational and rotational analysis of microbeams in nanobiomaterials using Galerkin Decomposition (GDM) and Differential Transform Methods (DTM) is presented. The dependency of cell migration and growth on nanoscaffold porosity and pore size architecture in tissue regeneration is governed by a dynamic model for the nonlinear vibration and rotation of the microbeams of nanobiomaterials and represented by a set of nonlinear partial differential equations. The solutions of the governing model are obtained by applying GDM and DTM and good agreement is achieved with numerical Runge-Kutta method (RK4). From the results, it is observed that an increase in Duffing term resulted in the increase of the frequency of the micro-beam. An increase in the foundation term also resulted in a corresponding increase in the frequency of the system for both free and forced dynamic responses. This study will enhance the application of tissue engineering in the regeneration of damaged human body tissues.
\end{abstract}

Mathematical Subject Classification: 35M86

Keywords: Nonlinear vibration, rotation, microbeams, nanobiomaterials, Galerkin Decomposition Method (GDM), Differential Transform Method (DTM)

\section{INTRODUCTION}

Cellular structured nanobiomaterials with extremely restrained micro-architectures have a wide range of applications which includes bone-substituting biomaterials in orthopaedics 1. In these applications, the size and size distribution of the biomaterials are important [2]. The production of these biomaterials has been enabled by applying additive manufacturing techniques in engineering principles to produce 
one or multiple types of unit cells. One of the most recent applications of the unit cell in the production of porous biomaterials is the diamond lattice unit cell [1, 3. In the application of additively manufacturing to porous titanium implants as replacements for bone, it is observed that the excellent biocompatible properties of titanium are preserved, which shows that the stiffness of titanium is quite small when compared with that of the natural bones [4]. The porosity and permeability of materials have been linked to fractal dimensions through imbibition model for petro-physical applications 5].

Nanoporous biomaterials such as Metal-Organic Frameworks (MOFs) consist of metal ions joined by organic connective ligands which have unique chemical and physical characteristics. The application of MOFs in biological systems, drug delivery, material science, and nanotechnology is being explored [2]. The importance and applications of biomaterials with more recent findings of smart biomaterials cannot be overemphasized, as uses are being found in the medicine and healthcare sector; as implants for body organ replacements, tissue regeneration, drug delivery systems, medical devices, and immune engineering 6 6].

Mathematical models for size dependent dynamics of biomaterials have been developed for three-layered beams based on the hypothesis of the Grigolyuk-Chulkov and the modified couple stress theory. The governing model and its boundary/initial conditions for beam displacement are applied for motion of layers' beams on the micro and nano-scales 10. Nanobeams with axially immovable ends and the geometrical nonlinearity caused by mid-plane stretching are considered in the developed model. In the Euler-Bernoulli beam model, the nano-device dynamic equation of motion is applied in the model kinematics [11]. The mathematical models of the nanoparticle are also affected by size and architecture, as shown in the normalized center deflections obtained in the study of size dependent composite laminated skew Mindlin plate. The Raleigh-Ritz method was applied to obtain a numerical solution to the model and it was observed that the normalized center deflections are always smaller than those obtained by the classical one $[12,13$. The mechanisms of the nanoparticles will aid the understanding of cell and nanoparticle size dependent toxicity. The electromechanical response of a nanostructure is observed to be influenced by the size of its element. This property of the nanostructure has been exhibited and it is further shown that the stability of the nanotweezers will be affected by the element size 14 . In the modeling of size effects of nanobeams, Reddy's shear deformation beam theory was applied to vibration characteristics of functional graded piezoelectric (FGP) nanobeams. Eringen's nonlocal elasticity theory was adopted to capture the small size effect. The obtained results showed that the applied Reddy's shear deformation model presented accurate frequency results of the FGP nanobeams [15].

Remarkably, mathematical models have provided the means to understand the physiochemical and physiological features of the behavior of nanomaterial in biological systems, as shown in the application of nanotechnology in inducing cytotoxic agents in cancer-nanomedicine [16], for predicting pore size distribution, and for the estimations of growth rates 17]. Nanomaterials of different shapes and sizes relate with cells in various ways, passively and actively. Recent studies on size-dependent 
effects of nanomaterials have been done with spherical nanoparticles but special consideration has been given to critical cellular interaction [13. In order to enhance the cellular interaction and nanoparticle internalization, it has been proposed that the best size for the nanoparticle is $50 \mathrm{~nm}$ 13], though most experimental data show that the approximate nanoparticle size preferred for cell internalization is $100 \mathrm{~nm}$. Hence nanoparticle size influences the endocytic pathway which is followed by cell internalization. The microtubules and the actin filaments are biological elements in the living cells which serve as block builders for functional nanomaterials and nanosurctures used for manufacturing nature - inspired small-scale devices or systems 18.

Analytical solutions have been obtained for wave dispersion in anisotropic doublycurved nanoshells. The governing equation for the formulation was based on Hamilton's principles 19. In a related study of free vibration of piezoelectric nanotubes, Hamilton's principles were applied to develop the governing equation and the enthalpy energy 20 22]. The effects of size as well as the geometrical and electromechanical effects on the nanotube and their effects on natural frequency of the vibration of the piezoelectric nanotubes were investigated. An explicit expression obtained for mechanical properties of nanoporous biomaterials. The expression was made in terms of the pore size from the lattice structure of the refined truncated cube by applying additive manufacturing in order to obtain appropriate mechanical properties [23]. The effectiveness, robustness and applicability of analytical solutions to complex problems cannot be overemphasized and this makes the analytical solution of the present problem of utmost importance. The study is focused on investigating the problems of nonlinear vibrational and rotational analysis of microbeams in nanobiomaterials using Galerkin Decomposition and Differential Transform Methods.

The developed nonlinear differential equations which describe the nonlinear vibrational and rotational analysis of microbeams in nanobiomaterials do not have closed form solutions, hence special analytical techniques are applied to obtain the solution: the Galerkin Decomposition Methoid and the Differential Transform Method. The Galerkin Decomposition Method is a numerical method which has proved to be effective in various applications, which include nonlinear elastic dynamics of a clamped laminated composite 24 and solution of wide range of weighted residual problems using Galerkin's Method [25. Galerkin's method has also been applied to nonlinear vibrational problems in carbon nanotubes applications [26], and heat transfer problems for temperature-dependent thermal conductivity of a porous fin in 27]. Galerkin's method has been combined with other methods to obtain more efficient results, such as the Variational methods, in solving engineering problems in nonlinear ordinary differential equations 28] and Petrov-Galerkin methods for nonlinear systems without monotonicity 29].

In this study, the solution obtained by the Galerkin Decomposition Method is compared with the solutions of the differential transform method. The differential transform method (DTM) is an approximate analytical method for solving linear and nonlinear ordinary and partial differential equations. It was proposed by Zhou in 1986 [30. The DTM has been applied in free and forced convection flow about inclined surfaces in porous media [31, 32], in Newtonian and non-Newtonian nanofluids flow 
analysis 33, and nonlinear ordinary differential matrix equations 34. DTM has proved to be effective when compared with the Adomian Decomposition Method $(\mathrm{ADM})$ and with the Variation Iteration Method (VIM) and Homotopy Perturbation Method (HPM) 35, 36]. The DTM has some drawbacks which have been overcome by combining it with Laplace transform in studies for nonlinear Duffing oscillator with damping effect 37] and for non-linear oscillators by applying the multi-step differential transform method 38. Further works on vibration theories and applications have also been presented 39,40].

Therefore the main objective of this current study is to investigate the problem of nonlinear vibrational and rotational analysis of microbeams in nanobiomaterials using Galerkin Decomposition and the Differential Transform Methods. In replacing damaged human body tissues, novel approaches are emerging in tissues engineering to regenerate damaged tissues and the major elements in these approaches are the migration and growth of cells. These elements depend on the porosity of the nanoscaffolds and the pore size architecture of the cell. In order to investigate this dependency of the migration and growth of cell on pore size, the nonlocal strain gradient theory of elasticity is applied to develop the dynamic model for the nonlinear vibration and rotation of the microbeams made of nanobiomaterials. This dynamic model is a set of nonlinear partial differential equations whose solutions require special analytic techniques. The solutions are obtained by applying Galerkin Decomposition Method or the Differential Transform Method. The effects of modal number on steady state response, the effect of duffing term on stability response of the microbeam, the effect of elastic foundations on microbeam stability response, and the effect of elastic foundation on microbeam free and forced dynamic responses are then investigated.

The paper is organized into five sections. The first one, i.e., the present section considers the preliminary results and outlines the problem to be solved. Section 2 is devoted to the equations of motion which have a strongly non-linear character. The solution algorithm is detailed in Section 3 where analytical approximations are devised for the unknown quantities. The numerical results are evaluated and discussed in Section 4. Our conclusions are presented in Section 5 which is a short summary of our results. The last section is a Nomenclature. The readers are advised to refer to this section for the fundamental notations.

\section{Formulating the equation of motion}

The degree of freedom of system for the microbeam's unit cell as well as the imposed boundary conditions are represented by Figures 1 and 2 In this present study, the biological system nanoporous microbeam is assumed to include the lattice structure of the refined truncated cube. With repeated cells, the unit cell is surrounded by truncated cubes and hence, results in each membrane of refined truncated cube. Consequently, analyzing a membrane of refined truncated cube is sufficient to obtain the mechanical response of the unit cell. If $\boldsymbol{\eta}_{2}=1, \boldsymbol{\eta}_{1}=\boldsymbol{\eta}_{3}$ and $\boldsymbol{\eta}_{4}=\boldsymbol{\eta}_{5}=\boldsymbol{\eta}_{6}=0$ it follows from Figure 1 that the point $a_{1}$ (the vertices of links $a_{1} b_{1}, a_{1} b_{2}, a_{1} b_{3}, a_{1} b_{4}$ - the last three links are, however, not represented in Figure 1) displaces downwards by unity. 


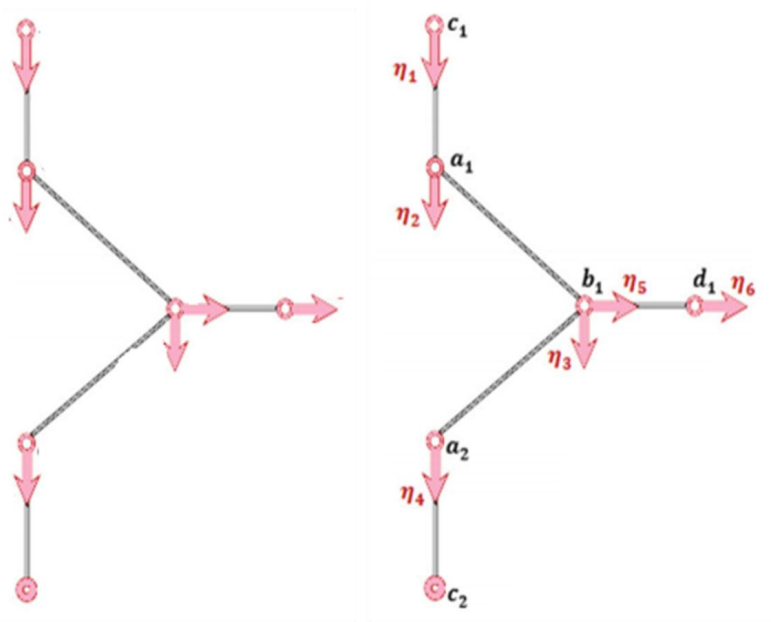

Figure 1. Cell unit with its degree of freedom

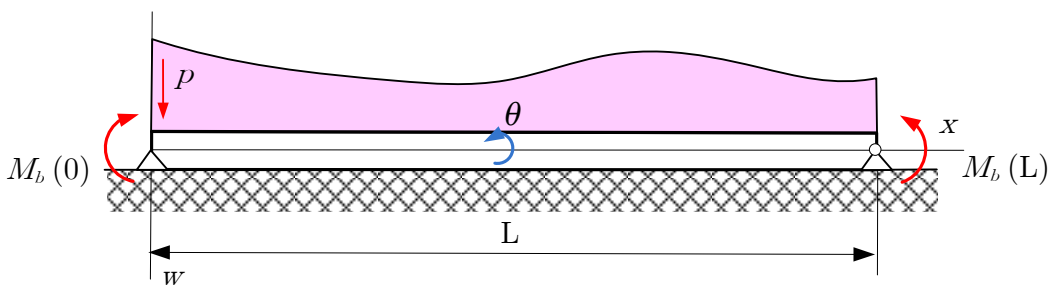

Figure 2. The system condition considered (pinned-pinned)

According to the refined hyperbolic shear deformable beam model proposed by Sahmani [41], the governing equations that capture the deflection and rotation are as follows:

$$
\begin{gathered}
\xi_{1} \frac{\partial^{4} w}{\partial x^{4}}-\xi_{2} \frac{\partial^{3} \theta}{\partial x^{3}}-\xi_{3} \frac{\partial^{6} w}{\partial x^{6}}+\xi_{4} \frac{\partial^{5} \theta}{\partial x^{5}}-p+\mu^{2} \frac{\partial^{2} p}{\partial x^{2}}-\xi_{5} \frac{\partial^{2} w}{\partial x^{2}}+ \\
+\mu^{2} \xi_{6} \frac{\partial^{2} w}{\partial x^{2}}+\mu^{2} \xi_{5} \frac{\partial^{4} w}{\partial x^{4}}+\xi_{7} \frac{\partial^{2} w}{\partial t^{2}}-\left(\mu^{2} \xi_{7}+\xi_{8}\right) \frac{\partial^{4} w}{\partial x^{2} \partial t^{2}}+\xi_{9} \frac{\partial^{6} w}{\partial x^{4} \partial t^{2}}- \\
-\xi_{10} \frac{\partial^{3} w}{\partial x \partial t^{2}}+\mu^{2} \xi_{10} \frac{\partial^{5} w}{\partial x^{3} \partial t^{2}}=0 \\
\xi_{1} \frac{\partial^{3} w}{\partial x^{3}}-\xi_{11} \frac{\partial^{2} \theta}{\partial x^{2}}+\xi_{12} \theta-\xi_{12} \frac{\partial^{3} w}{\partial x \partial t^{2}}-\xi_{13} \frac{\partial^{2} w}{\partial t^{2}}=0 .
\end{gathered}
$$


In this work, a nonlinear elastic foundation term will be incorporated. This makes the fully coupled governing equation strongly nonlinear as presented below:

$$
\begin{gathered}
R_{1}(x, t)=\xi_{1} \frac{\partial^{4} w}{\partial x^{4}}-\xi_{2} \frac{\partial^{3} \theta}{\partial x^{3}}-\xi_{3} \frac{\partial^{6} w}{\partial x^{6}}+\xi_{4} \frac{\partial^{5} \theta}{\partial x^{5}}-p+\mu^{2} \frac{\partial^{2} p}{\partial x^{2}}-\xi_{5} \frac{\partial^{2} w}{\partial x^{2}}+ \\
+\mu^{2} \xi_{6} \frac{\partial^{2} w}{\partial x^{2}}+\mu^{2} \xi_{5} \frac{\partial^{4} w}{\partial x^{4}}+\xi_{7} \frac{\partial^{2} w}{\partial t^{2}}-\left(\mu^{2} \xi_{7}+\xi_{8}\right) \frac{\partial^{4} w}{\partial x^{2} \partial t^{2}}+\xi_{9} \frac{\partial^{6} w}{\partial x^{4} \partial t^{2}}- \\
-\xi_{10} \frac{\partial^{3} w}{\partial x \partial t^{2}}+\mu^{2} \xi_{10} \frac{\partial^{5} w}{\partial x^{3} \partial t^{2}}+k_{1} w+k_{2} w^{3}=0 \\
R_{2}(x, t)=\xi_{1} \frac{\partial^{3} w}{\partial x^{3}}-\xi_{11} \frac{\partial^{2} \theta}{\partial x^{2}}+\xi_{12} \theta-\xi_{12} \frac{\partial^{3} w}{\partial x \partial t^{2}}-\xi_{13} \frac{\partial^{2} w}{\partial t^{2}}=0
\end{gathered}
$$

The non-linear differential equations (2) are associated with the following boundary conditions valid for pinned-pinned beams 40]:

$$
\begin{gathered}
w(0, t)=w^{\prime \prime}(0, t)=0, \\
w(L, t)=w^{\prime \prime}(L, t)=0 .
\end{gathered}
$$

The initial conditions are of the form

$$
\begin{gathered}
w(t=0)=a=\lim _{t \rightarrow 0} \frac{\bar{w}}{10^{-6} m} \cos \bar{\omega} t=W_{\max } \cos \bar{\omega} t, \quad \dot{w}(t=0)=0, \\
\theta(t=0)=b=\lim _{t \rightarrow 0} b \cos \bar{\omega} t, \quad \dot{\theta}(t=0)=0 .
\end{gathered}
$$

Here, the initial deflection and rotation of the microbeam, which are $a$ and $b$, tend to zero 41. Equation 2 will be solved using Galerkin Decomposition (DG) and Differential Transform Method (DTM) in order to obtain the dynamic response and rotation of the system under consideration.

\section{Models AND SOLUTiOnS}

3.1. Application of the Galerkin decomposition. The Galerkin Decomposition method is applied to convert the governing partial differential equations into ordinary differential equations using an appropriate shape function that satisfies the boundary conditions. This approach is expressed as

$$
\int_{0}^{L} R_{i}(x, t) \varphi(x) d x=0, \quad(i=1,2)
$$

where

$$
w=w(x, t)=T(t) \varphi(x), \quad \text { and } \quad \theta=\theta(x, t)=J(t) \varphi(x)
$$


while $R_{1}$ and $R_{2}$ are given by (2). Substituting them into (4) yields

$$
\begin{gathered}
\int_{0}^{L}\left(\xi_{1} \frac{\partial^{4} w}{\partial x^{4}}-\xi_{2} \frac{\partial^{3} \theta}{\partial x^{3}}-\xi_{3} \frac{\partial^{6} w}{\partial x^{6}}+\xi_{4} \frac{\partial^{5} \theta}{\partial x^{5}}-p+\mu^{2} \frac{\partial^{2} p}{\partial x^{2}}-\xi_{5} \frac{\partial^{2} w}{\partial x^{2}}+\right. \\
+\mu^{2} \xi_{6} \frac{\partial^{2} w}{\partial x^{2}}+\mu^{2} \xi_{5} \frac{\partial^{4} w}{\partial x^{4}}+\xi_{7} \frac{\partial^{2} w}{\partial t^{2}}-\left(\mu^{2} \xi_{7}+\xi_{8}\right) \frac{\partial^{4} w}{\partial x^{2} \partial t^{2}}+\xi_{9} \frac{\partial^{6} w}{\partial x^{4} \partial t^{2}}- \\
\left.-\xi_{10} \frac{\partial^{3} w}{\partial x \partial t^{2}}+\mu^{2} \xi_{10} \frac{\partial^{5} w}{\partial x^{3} \partial t^{2}}+k_{1} w+k_{2} w^{3}\right) \varphi(x) d x=0 \\
\int_{0}^{L}\left(\xi_{1} \frac{\partial^{3} w}{\partial x^{3}}-\xi_{11} \frac{\partial^{2} \theta}{\partial x^{2}}+\xi_{12} \theta-\xi_{12} \frac{\partial^{3} w}{\partial x \partial t^{2}}-\xi_{13} \frac{\partial^{2} w}{\partial t^{2}}\right) \varphi(x) d x=0 .
\end{gathered}
$$

from where by inserting (5) we have

$$
\begin{gathered}
\int_{0}^{L}\left(\xi_{1} \frac{\partial^{4} T(t) \varphi(x)}{\partial x^{4}}-\xi_{2} \frac{\partial^{3} J(t) \varphi(x)}{\partial x^{3}}-\xi_{3} \frac{\partial^{6} T(t) \varphi(x)}{\partial x^{6}}+\xi_{4} \frac{\partial^{5} J(t) \varphi(x)}{\partial x^{5}}-\right. \\
-p+\mu^{2} \frac{\partial^{2} p}{\partial x^{2}}-\xi_{5} \frac{\partial^{2} T(t) \varphi(x)}{\partial x^{2}}+\mu^{2} \xi_{6} \frac{\partial^{2} T(t) \varphi(x)}{\partial x^{2}}+\mu^{2} \xi_{5} \frac{\partial^{4}(T(t) \varphi(x))}{\partial x^{4}}+ \\
+\xi_{7} \frac{\partial^{2} T(t) \varphi(x)}{\partial t^{2}}-\left(\mu^{2} \xi_{7}+\xi_{8}\right) \frac{\partial^{4} T(t) \varphi(x)}{\partial x^{2} \partial t^{2}}+\xi_{9} \frac{\partial^{6} T(t) \varphi(x)}{\partial x^{4} \partial t^{2}}-\xi_{10} \frac{\partial^{3} T(t) \varphi(x)}{\partial x \partial t^{2}}+ \\
\left.+\mu^{2} \xi_{10} \frac{\partial^{5} T(t) \varphi(x)}{\partial x^{3} \partial t^{2}}+k_{1} T(t) \varphi(x)+k_{2}(T(t) \varphi(x))^{3}\right) \varphi(x) d x=0
\end{gathered}
$$

and

$$
\begin{aligned}
& \int_{0}^{L}\left(\xi_{1} \frac{\partial^{3} T(t) \varphi(x)}{\partial x^{3}}-\xi_{11} \frac{\partial^{2} J(t) \varphi(x)}{\partial x^{2}}+\xi_{12} J(t) \varphi(x)-\right. \\
& \left.-\xi_{12} \frac{\partial^{3} T(t) \varphi(x)}{\partial x \partial t^{2}}-\xi_{13} \frac{\partial^{2} J(t) \varphi(x)}{\partial t^{2}}\right) \varphi(x) d x=0 .
\end{aligned}
$$

By introducing new notations equations, (7) and (8) can be manipulated into the following simple forms

$$
\begin{gathered}
M_{1} \ddot{T}+K_{1} T+K_{2} J+V T^{3}=F, \\
M_{2} \ddot{J}+K_{12} J+K_{21} T=0,
\end{gathered}
$$

where

$$
\begin{aligned}
M_{1}=\int_{0}^{L}\left(\xi_{7} \varphi(x)-\left(\mu^{2} \xi_{7}+\xi_{8}\right) \frac{d^{2} \varphi(x)}{d x^{2}}\right. & +\xi_{9} \frac{d^{4} \varphi(x)}{d x^{4}}+ \\
& \left.\quad+\xi_{10} \frac{d \varphi(x)}{d x}+\mu^{2} \xi_{10} \frac{d^{3} \varphi(x)}{d x^{3}}\right) \varphi(x) d x
\end{aligned}
$$




$$
\begin{gathered}
K_{1}=\int_{0}^{L}\left(\xi_{1} \frac{d^{4} \varphi(x)}{d x^{4}}-\xi_{3} \frac{d^{6} \varphi(x)}{d x^{6}}-\xi_{5} \frac{d^{2} \varphi(x)}{d x^{2}}+\right. \\
\left.+\mu^{2} \xi_{6} \frac{d^{2} \varphi(x)}{d x^{2}}+\mu^{2} \xi_{5} \frac{d^{4} \varphi(x)}{d x^{4}}+k_{1} \varphi(x)\right) \varphi(x) d x \\
K_{2}=\int_{0}^{L}\left(-\xi_{2} \frac{d^{3} J(t) \varphi(x)}{d x^{3}}+\xi_{4} \frac{d^{5} J(t) \varphi(x)}{d x^{5}}\right) \varphi(x) d x \\
V=\int_{0}^{L} k_{2}(\varphi(x))^{3} \varphi(x) d x
\end{gathered}
$$

and

$$
\begin{gathered}
M_{2}=\int_{0}^{L}\left(\xi_{13} \varphi(x)\right) \varphi(x) d x, \\
M_{21}=-\int_{0}^{L} \xi_{12} \frac{d \varphi(x)}{d x} \varphi(x) d x=0, \\
K_{12}=\int_{0}^{L}\left(\xi_{11} \frac{d^{2} \varphi(x)}{d x^{2}}+\xi_{12} \varphi(x)\right) \varphi(x) d x, \\
K_{21}=\int_{0}^{L} \xi_{1} \frac{d^{3} \varphi(x)}{d x^{3}} \varphi(x) d x .
\end{gathered}
$$

Equation (9) is the desired system of ODE from the Galerkin decomposition of the PDEs which will be solved using DTM. However, the natural frequency and frequency ratio of the system may be obtained as

$$
\omega=\sqrt{\frac{K_{1}}{M_{1}}}
$$

and

which in an expanded form are given by

$$
\Omega=\frac{\omega_{n l}}{\omega}=\sqrt{1 \pm \frac{3 V W_{\max }^{2}}{4 K_{1}}}
$$

$$
\begin{aligned}
& \omega= \\
& =\sqrt{\frac{\int_{0}^{L}\left(\xi_{1} \frac{d^{4} \varphi(x)}{d x^{4}}-\xi_{3} \frac{d^{6} \varphi(x)}{d x^{6}}-\xi_{5} \frac{d^{2} \varphi(x)}{d x^{2}}+\mu^{2} \xi_{6} \frac{d^{2} \varphi(x)}{d x^{2}}+\mu^{2} \xi_{5} \frac{d^{4} \varphi(x)}{d x^{4}}+k_{1} \varphi(x)\right) \varphi(x) d x}{\int_{0}^{L}\left(\xi_{7} \varphi(x)-\left(\mu^{2} \xi_{7}+\xi_{8}\right) \frac{d^{2} \varphi(x)}{d x^{2}}+\xi_{9} \frac{d^{4} \varphi(x)}{d x^{4}}+\xi_{10} \frac{d \varphi(x)}{d x}+\mu^{2} \xi_{10} \frac{d^{3} \varphi(x)}{d x^{3}}\right) \varphi(x) d x}},
\end{aligned}
$$




$$
\begin{aligned}
& \Omega=\frac{\omega_{n l}}{\omega}= \\
& =\sqrt{1 \pm \frac{3 W_{\max }^{2} \int_{0}^{L} k_{2}(\varphi(x))^{3} \varphi(x) d x}{4 \int_{0}^{L}\left(\xi_{1} \frac{d^{4} \varphi(x)}{d x^{4}}-\xi_{3} \frac{d^{6} \varphi(x)}{d x^{6}}-\xi_{5} \frac{d^{2} \varphi(x)}{d x^{2}}+\mu^{2} \xi_{6} \frac{d^{2} \varphi(x)}{d x^{2}}+\mu^{2} \xi_{5} \frac{d^{4} \varphi(x)}{d x^{4}}+k_{1} \varphi(x)\right) \varphi(x) d x}},
\end{aligned}
$$

where $\varphi=\sin \frac{n \pi}{L} x$ for the considered pinned-pinned beam, $n$ is the modal number and $F$ is the Galerkin form of $p$-applying Galerkin decomposition to $p$ yields $F$.

\subsection{Analytical solution to the developed models and the basic concepts of} the differential transform method (DTM). Due to the presence of a nonlinearity in the derived coupled governing equation of motion, a method capable of transforming differential equations into another domain with a robust and easy way of inversion is required. The differential transform method (DTM) possesses this attribute. DTM maps a governing equation into an algebraic domain and then obtains an inversion using a series summation method. This approximate analytical method generates a solution with the controlling parameters adequately conserved. The recursive relations that constitute DTM for transforming differential equation into the desired form are shown in Table 1. As regards the notations used in this table we refer the reader to [30] and 34 which detail the way they should be applied. By applying this scheme to equations (9) we obtain the required analytical solutions of the governing equations.

Table 1. Recursive relations for the Differential Transform Method (DTM)

$$
\begin{gathered}
Z(t)=U(t) \pm V(t), \quad Z(k)=U(k) \pm V(k) ; \\
Z(t)=\infty U(t), \quad Z(k)=\infty U(k) ; \\
Z(t)=\frac{d U(t)}{d t}, \quad Z(k)=(k+1) U[k+1] ; \\
Z(t)=\frac{d^{2} U(t)}{d t^{2}}, \quad Z(k)=(k+1)(k+2) U[k+2] ; \\
Z(t)=\frac{d^{m} U(t)}{d t^{m}}, \quad Z(k)=(k+1)(k+2) \cdots U[k+m]=\frac{(k+m) !}{k !} U[k+m] ; \\
Z(t)=U(t) * V(t), \quad Z(k)=\sum_{\ell=0}^{K} V(L) U[K-\ell] ; \\
Z(t)=t^{m}, \quad Z(k)=\delta(k-m) .
\end{gathered}
$$

After applying the scheme in Table 1 to equations $(9)$ we have

$$
\begin{aligned}
M_{1}(k+1)(k+2) T_{k+2}+ & K_{1} T_{k}+K_{2} J_{k}+ \\
& +V \sum_{q=0}^{k}\left(\sum_{l=0}^{q} T_{l} T_{q-l} T_{k-q}\right)-\frac{F \omega^{k} \sin (1 / 2 k \pi)}{k !}=0
\end{aligned}
$$


and

$$
M_{2}(k+1)(k+2) J_{k+2}+K_{12} J_{k}+K_{21} T_{k}=0,
$$

where with regard to the transformed initial conditions

$$
T_{0}=a, \quad T_{1}=0, \quad J_{0}=b \quad \text { and } \quad J_{1}=0 .
$$

Performing the iteration steps on equations $(16)$ and $(17)$ by utilizing equations 18 leads to the following solutions for $T_{2}, J_{2}, \ldots, T_{7}, J_{7}$ :

$$
\begin{aligned}
& T_{2}=-\frac{V a^{3}+K_{1} a+K_{2} b}{2 M_{1}}, \\
& J_{2}=-\frac{K_{12} b+K_{21} a}{2 M_{2}}, \\
& T_{3}=\frac{F \omega}{16 M_{1}}, \\
& J_{3}=0 \text {, } \\
& T_{4}=\frac{1}{24 M_{1}^{2} M_{2}}\left(3 M_{2} V^{2} a^{5}+4 K_{1} M_{2} V a^{3}+3 K_{2} M_{2} V a^{2} b+K_{1}^{2} M_{2} a+\right. \\
& \left.+K_{1} K_{2} M_{2} b+K_{12} K_{2} M_{1} b+K_{2} K_{21} M_{1} a\right), \\
& J_{4}=\frac{K_{21} M_{2} V a^{3}+K_{1} K_{21} M_{2} a+K_{12}^{2} M_{1} b+K_{12} K_{21} M_{1} a+K_{2} K_{21} M_{2} b}{24 M_{1} M_{2}^{2}}, \\
& T_{5}=-F \omega\left(M_{1} \omega^{2}+3 V a^{2}+K_{1}\right) / 120 M_{1}^{2} \\
& J_{5}=-K_{21} F \omega / 120 M_{1} M_{2}, \\
& T_{6}=-\frac{1}{720 M_{1}^{3} M_{2}^{2}}\left(27 M_{2}^{2} V^{3} a^{7}+51 K_{1} M_{2}^{2} V^{2} a^{5}+45 K_{2} M_{2}^{2} V^{2} a^{4} b+\right. \\
& +25 K_{1}^{2} M_{2}^{2} V a^{3}+42 K_{1} K_{2} M_{2}^{2} V a^{2} b+3 K_{12} K_{2} M_{1} M_{2} V a^{2} b+18 K_{2}^{2} M_{2}^{2} V a b^{2}+ \\
& +4 K_{2} K_{21} M_{1} M_{2} V a^{3}+K_{1}^{3} M_{2}^{2} a+K_{1}^{2} K_{2} M_{2}^{2} b+K_{1} K_{12} K_{2} M_{1} M_{2}+ \\
& \left.+2 K_{1} K_{2} K_{21} M_{1} M_{2} a+K_{12}^{2} K_{2} M_{1}^{2} b+K_{12} K_{2} K_{21} M_{1}^{2} a+K_{2}^{2} K_{21} M_{1} M_{2} b\right), \\
& J_{6}=-\frac{1}{720 M_{1}^{2} M_{2}^{3}}\left(3 K_{21} M_{2}^{2} V^{2} a^{5}+4 K_{1} K_{21} M_{2}^{2} V a^{3}+K_{12} K_{21} M_{1} M_{2} V a^{3}+\right. \\
& +3 K_{2} K_{21} M_{2}^{2} V a^{2} b+K_{1}^{2} K_{21} M_{2}^{2} a+K_{1} K_{12} K_{21} M_{1} M_{2} a+ \\
& +K_{1} K_{2} K_{21} M_{2}^{2} b+K_{12}^{3} M_{1}^{2} b+K_{12}^{2} K_{21} M_{1}^{2} a+2 K_{12} K_{2} K_{21} M_{1} M_{2} b+ \\
& \left.+K_{2} K_{21}^{2} M_{1} M_{2} a\right) \text {, } \\
& T_{7}=\frac{F \omega}{5040 M_{1}^{3} M_{2}}\left(M_{1}^{2} M_{2} \omega^{4}+3 M_{1} M_{2} V a^{2} \omega^{2}+69 M_{2} V^{2} a^{4}+K_{1} M_{1} M_{2} \omega^{2}+\right. \\
& \left.+66 K_{1} M_{2} V a^{2}+60 K_{2} M_{2} V a b+K_{1}^{2} M_{2}+K_{2} K_{21} M_{1}\right), \\
& J_{7}=\frac{K_{21} F \omega\left(M_{1} M_{2} \omega^{2}+3 M_{2} V a^{2}+K_{1} M_{2}+K_{12} M_{1}\right)}{5040 M_{1}^{2} M_{2}^{2}} .
\end{aligned}
$$


With $T_{0}, J_{0}, \ldots, T_{7}, J_{7}$ we have the following analytical approximations

$$
T(t)=\sum_{k=0}^{7} T_{k} t^{k}, \quad J(t)=\sum_{k=0}^{7} J_{k} t^{k} .
$$

Equations (31), in which the coefficients are given by $[18, \ldots,(30)$, are the desired analytical solutions for $T(t)$ and $J(t)$. In order to find the unknown deflections for a longer time history, the above technique is applied and the results obtained are utilized to analyze the dynamic behavior of the system in the present study. Furthermore, the computations require 16 iterations for achieving good accuracy.

\section{Results And Discussions}

In this study, the nonlinear vibration and rotation of microbeams in presented. The migration and growth of a cell depends on the porosity of the nanoscaffolds and the pore size architecture. In order to investigate this dependency of the cell migration and growth on pore size, the nonlocal strain gradient theory of elasticity is applied to develop a dynamic model for the nonlinear vibration and rotation of microbeams made of nanobiomaterials. This dynamic model, which is a set of coupled nonlinear ordinary differential equations, was solved by applying an approximate

Table 2. Validation of the Galerkin Decomposition Method with the Differential Transform Method

\begin{tabular}{|c|r|r|r|r|r|c|}
\hline Time & \multicolumn{3}{|c|}{ Deflection (nm) } & \multicolumn{3}{c|}{ Rotation (radian) } \\
\hline (Secs) & GDM & DTM & Residual & GDM & DTM & Residual \\
\hline \hline 1 & 0.3000 & 0.3000 & 0.0000 & 0.1000 & 0.1000 & 0.0000 \\
\hline 2 & 0.2384 & 0.2385 & 0.0001 & 0.0921 & 0.0921 & 0.0000 \\
\hline 3 & 0.0787 & 0.0790 & 0.0003 & 0.0711 & 0.0711 & 0.0000 \\
\hline 4 & -0.1150 & -0.1144 & 0.0006 & 0.0441 & 0.0440 & 0.0001 \\
\hline 5 & -0.2649 & -0.2645 & 0.0004 & 0.0198 & 0.0197 & 0.0001 \\
\hline 6 & -0.3104 & -0.3105 & 0.0001 & 0.0051 & 0.0050 & 0.0001 \\
\hline 7 & -0.2327 & -0.2335 & 0.0008 & 0.0025 & 0.0023 & 0.0002 \\
\hline 8 & -0.0628 & -0.0641 & 0.0013 & 0.0088 & 0.0087 & 0.0001 \\
\hline 9 & 0.1316 & -0.1302 & 0.0014 & 0.0173 & 0.0172 & 0.0001 \\
\hline 10 & 0.2732 & -0.2724 & 0.0008 & 0.0199 & 0.0199 & 0.0000 \\
\hline 11 & 0.3056 & 0.3059 & 0.0003 & 0.0110 & 0.1112 & 0.0002 \\
\hline 12 & 0.2160 & 0.2175 & 0.0015 & -0.0104 & -0.0100 & 0.0003 \\
\hline 13 & 0.0413 & 0.0435 & 0.0022 & -0.0398 & -0.0395 & 0.0003 \\
\hline 14 & -0.1482 & -0.1461 & 0.0021 & -0.0693 & -0.0690 & 0.0003 \\
\hline 15 & -0.2764 & -0.2753 & 0.0011 & -0.0901 & -0.0900 & 0.0001 \\
\hline 16 & -0.2912 & -0.2919 & 0.0007 & -0.0964 & -0.0965 & 0.0001 \\
\hline 17 & -0.1865 & -0.1889 & 0.0008 & -0.0874 & -0.0876 & 0.0002 \\
\hline 18 & -0.0046 & -0.0078 & 0.0032 & -0.0675 & -0.0678 & 0.0003 \\
\hline 19 & 0.1818 & 0.1790 & 0.0028 & -0.0446 & -0.0449 & 0.0003 \\
\hline 20 & 0.2980 & 0.2969 & 0.0011 & -0.0272 & -0.0272 & 0.0000 \\
\hline
\end{tabular}


solution method; Galerkin Decomposition Method and an approximate analytical approach the Differential Transform Method. The results are shown in Table 2 and an excellent agreement is established between them.

The effects of modal number on steady state response are shown in Figures 3 . 6. This analysis is vital as it shows clearly the locations of nodes and anti-nodes. Based on the results obtained, an increase in modal number increases the number

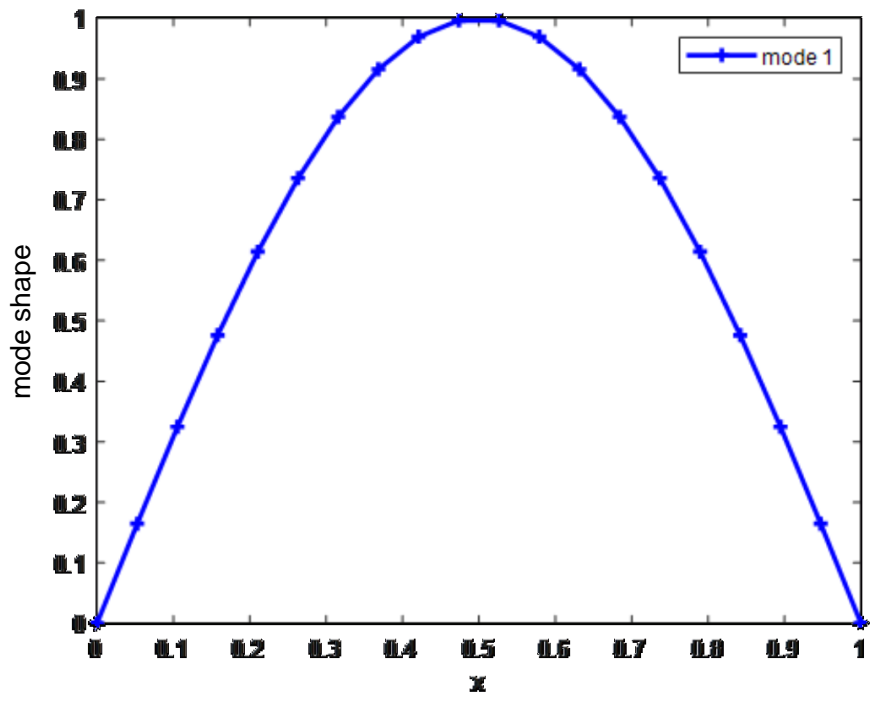

Figure 3. Steady state response for mode 1

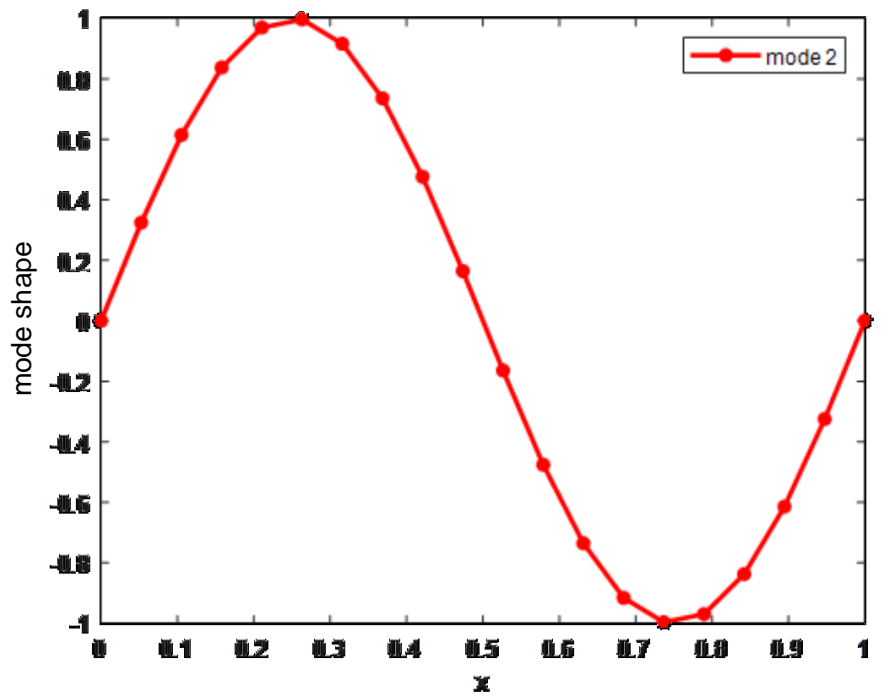

Figure 4. Steady state response for mode 2 


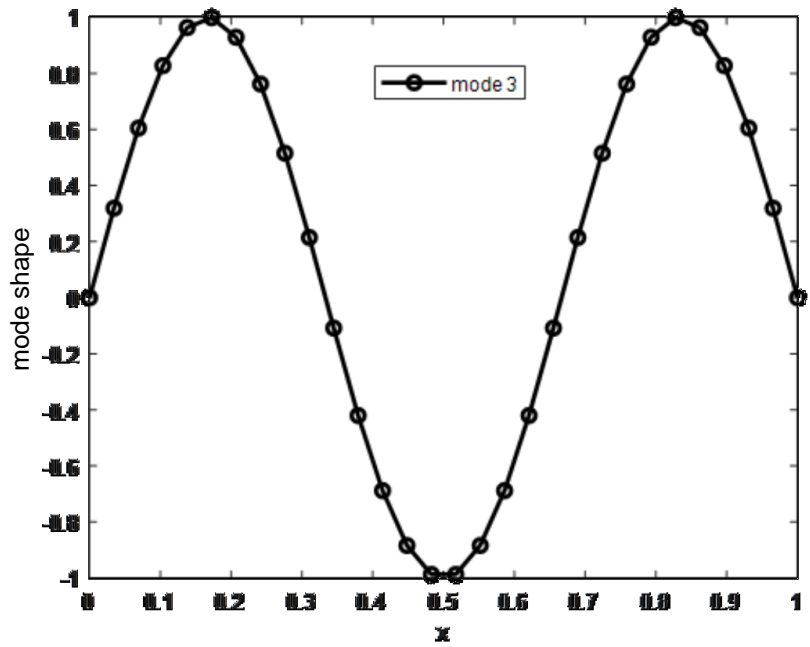

Figure 5. Steady state response for mode 3

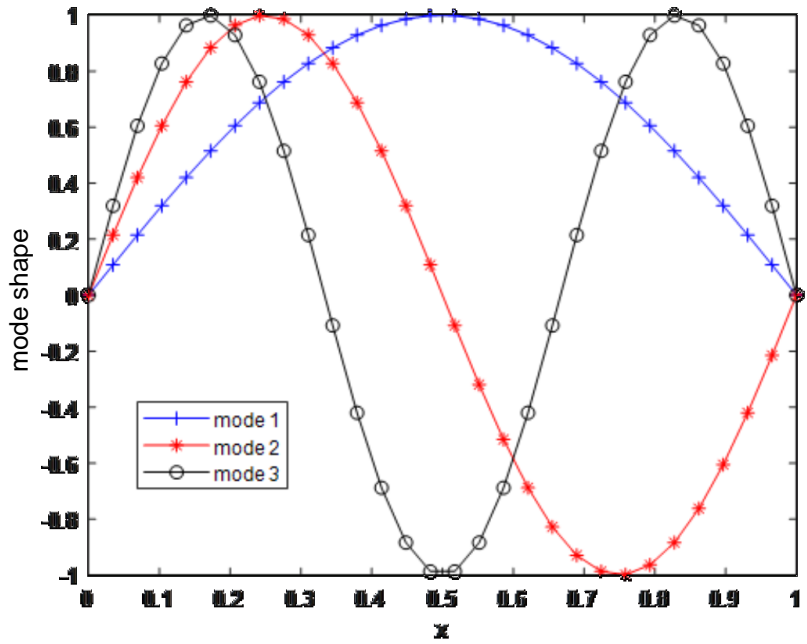

Figure 6. Super-imposed steady state responses

of nodes and anti-nodes. The locations of anti-nodes are very important during the system's vibration that may tend to resonance as reducing disturbances at these point automatically reduces vibration throughout the entire beam length.

The effect of the Duffing term on micro $W_{\max }$ is shown in Figure 7. From the plot, it is obvious that when the system's deflection is low, the nonlinear term possesses negligible impact. However, at very large amplitudes, an increase in the Duffing term increases the dimensionless frequency of the nano-porous micro-beam. Figure 8 shows 


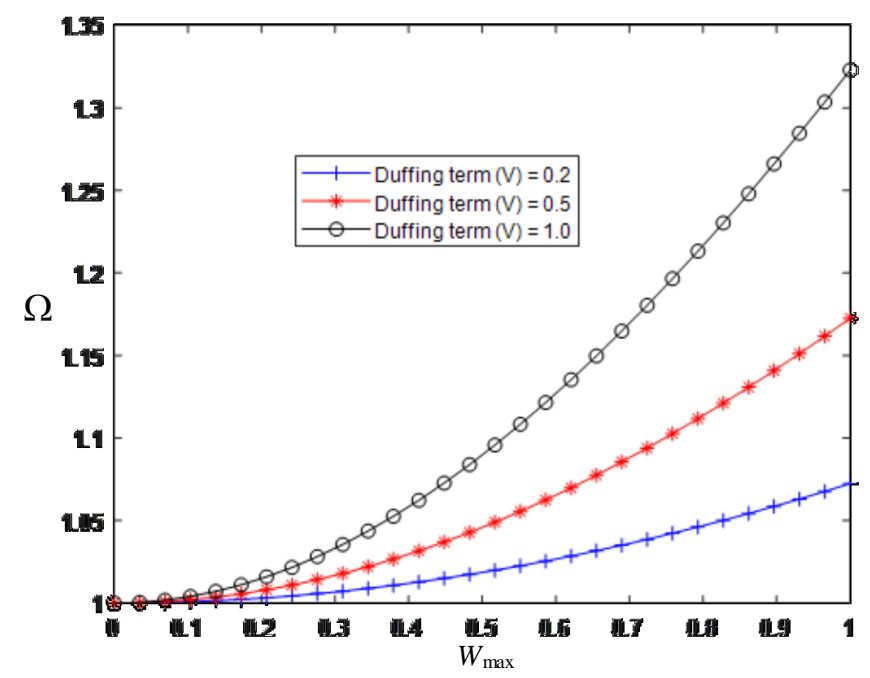

Figure 7. The effect of the nonlinear term on $W_{\max }$

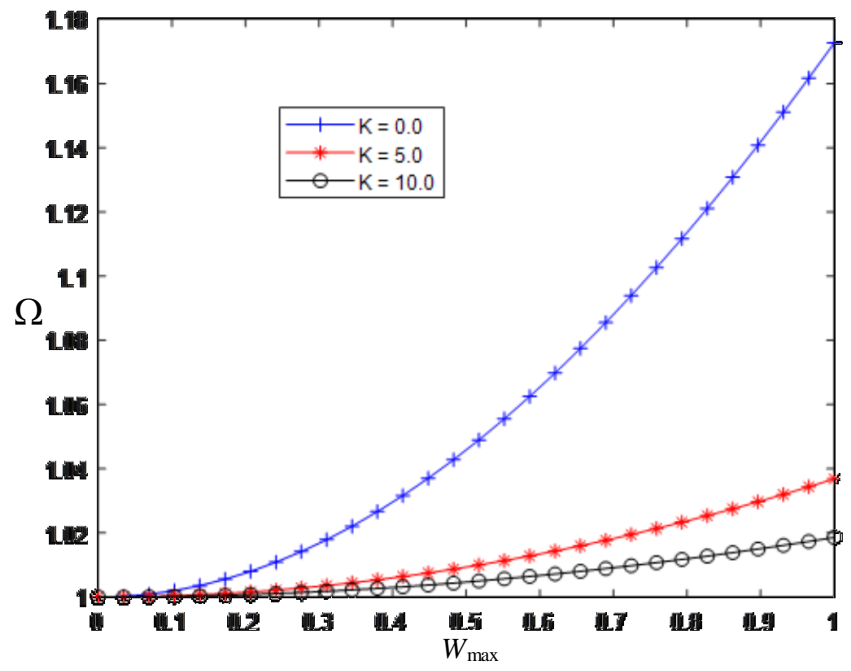

Figure 8. The effect of the foundation term on $W_{\max }$

the effect of elastic foundation term $\mathrm{m}$ (also referred to as the linear Winkler coefficient $k=k_{1}$ ) on on $W_{\max }$. From the plot, it is observed that when the micro-beam is foundation free, it gives a very large frequency ratio. This may result in instable behavior. However, when the elastic foundation parameter is introduced, the frequency ratio starts to decay even for high values of deflection. This reiterates the importance of an elastic foundation. Figures 7 and 8 are included in the study for monitoring the resonance of the microbeam. 


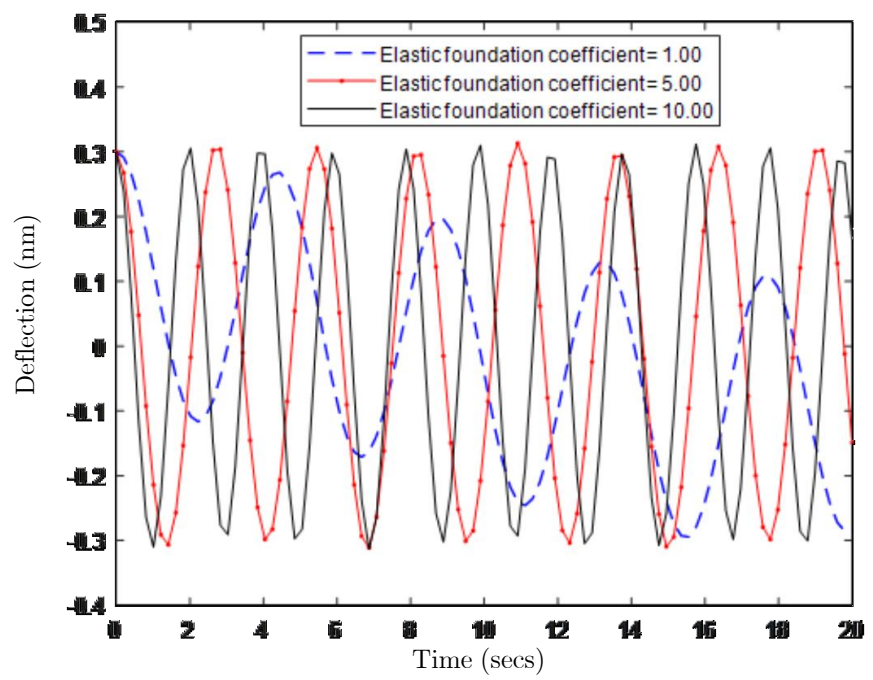

Figure 9. Effect of the foundation term on the free dynamic response

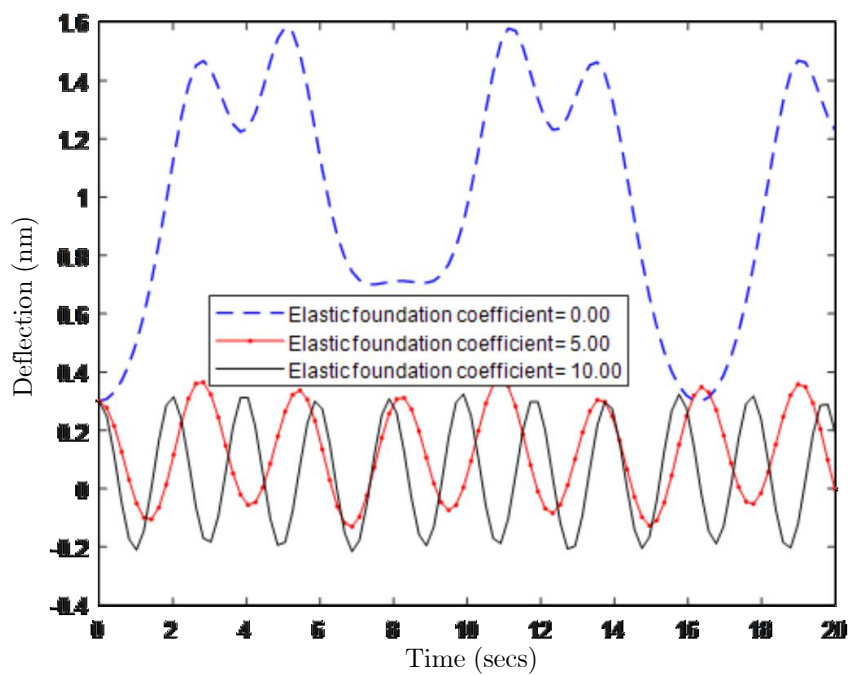

Figure 10. Effect of foundation term on the forced dynamic response

\section{Conclusion}

In this study, the nonlinear vibrational and rotational analysis of microbeams in nanobiomaterials using Galerkin Decomposition and the Differential Transform Method has been presented. The degeneration of human body tissues caused by congenital defects, diseases, trauma, etc. which were not replaced in times past, can now be 
replaced today with the novel approaches emerging in tissue engineering to regenerate such damaged tissues even after been replaced. The major element in one of the novel approaches is the migration and growth of cells, which depends on the porosity of the nanoscaffolds and the pore size architecture. In order to investigate this dependency of the cell migration and growth on pore size, the nonlocal strain gradient theory of elasticity is applied to develop a dynamic model for the nonlinear vibration and rotation of the microbeams made of nanobiomaterials. This dynamic model, which is a set of coupled nonlinear ordinary differential equations, was solved by applying a decomposition scheme - Galerkin Decomposition Method and an approximate analytical technique, the Differential Transform Method. Good agreement is established between the solutions. The effects of the modal number on the steady state response, the effect of the Duffing term on stability response on microbeam, the effect of the elastic foundations on the stability response of the microbeam, and the effect of the elastic foundation on the free and forced dynamic responses of the microbeam were investigated. It is observed that an increase in the modal number increases the number of the nodes and anti-nodes. During system vibration that may tend to resonance, the increased anti-nodes reduce disturbances at these nodal points which automatically reduces the vibration in the entire beam length. An increase in Duffing term also resulted in the increases of the dimensionless frequency of the nano-porous micro-beam. When the elastic foundation is introduced and increased, there is a decrease in the frequency ration of the microbeam. And for the free and forced dynamic responses, an increase in the foundation term increases the frequency of the system for both conditions. This study will enhance a parametric study in vibration and rotation of nanobiomaterials and the application of tissue engineering to regenerate damaged tissues in the human body.

\section{Nomenclature}

\begin{tabular}{|c|c|c|c|}
\hline & Latin notations & & \\
\hline$a_{1} b_{1}$, & Links - see Figure 1 & $t$ & Time \\
\hline$A$ & Area $\square$ & $T$ & Temporal rotation \\
\hline E & Modulus of elasticity & $V$ & Duffing term \\
\hline$F$ & Galerkin force function & $w$ & Deflection \\
\hline$G$ & Shear modulus of elasticity & $W_{\max }$ & Maximum dimensionless deflection \\
\hline$I$ & Moment of inertia & $\bar{w}$ & Deflection \\
\hline$J$ & Temporal rotation & $x$ & Independent variable \\
\hline$k_{1}$ & Linear Winkler coefficient & & Greek notations \\
\hline$k_{2}$ & Non-linear foundation coefficient & $\theta$ & Rotation \\
\hline$K$ & Stiffness & $\mu$ & Nonlocal term \\
\hline$L$ & Length of the microbeam & $\xi_{1}$ & Flexural term \\
\hline$M$ & Mass & $\xi_{2, \ldots, 13}$ & Known coefficients 41 \\
\hline$M_{b}$ & Bending moment & $\varphi$ & Shape function \\
\hline$n$ & Nodal number & $\omega$ & Natural frequency \\
\hline$p$ & Distributed load & $\omega_{n \ell}$ & Non-linear frequency \\
\hline$R$ & Galerkin Function & $\Omega$ & Frequency ratio \\
\hline
\end{tabular}




\section{REFERENCES}

1. O. V. Salata. "Applications of nanoparticles in biology and medicine." Journal of Nanobiotechnology, 2, (May 2004), pp. 1-6. DOI: 10.1186/1477-3155-2-3.

2. S. Beg, M. Rahman, A. Jain, S. Saini, P. Midoux, C. Pichon, F. J. Ahmad, and S. Akhter. "Nanoporous metal organic frameworks as hybrid polymer-metal composites for drug delivery and biomedical applications." Drug Discovery Today, 22(4) (2017), pp. 625 -637. DOI: 10.1016/j.drudis.2016.10.001.

3. S. M. Ahmadi, G. Campoli, S. Amin Yavari, B. Sajadi, R. Wauthle, J. Schrooten, H. Weinans, and A. A. Zadpoor. "Mechanical behavior of regular opencell porous biomaterials made of diamond lattice unit cells." Journal of the Mechanical Behavior of Biomedical Materials, 34, (2014), pp. 106 -115. DOI: https://doi.org/10.1016/j.jmbbm.2014.02.003.

4. R. Hedayati, S. Amin Yavari, and A. A. Zadpoor. "Fatigue crack propagation in additively manufactured porous biomaterials." Materials Science and Engineering: $C$, 76, (2017), pp. 457 -463. DOI: 10.1016/j.msec.2017.03.091.

5. A. Mumuni and P. Michal. "A mathematical determination of the pore size distribution and fractal dimension of a porous sample using spontaneous imbibition dynamics theory." Journal of Petroleum Exploration and Production Technology, 9, (2019), pp. 427 -435. DOI: 10.1007/s13202-018-0477-9.

6. P. S. Kowalski, C. Bhattacharya, S. Afewerki, and R. Langer. "Smart Biomaterials: Recent Advances and Future Directions." ACS Biomaterials Science and Engineering, 4(11), (2018). DOI: 10.1021/acsbiomaterials.8b00889.

7. D. Caccavo. "An overview on the mathematical modeling of hydrogels' behavior for drug delivery systems." International Journal of Pharmaceutics, 560, (2019), pp. 175 -190. DOI: 10.1016/j.ijpharm.2019.01.076.

8. M. E. Furth, A. Atala, and Mark E. Van Dyke. "Smart biomaterials design for tissue engineering and regenerative medicine." Biomaterials, 28(34), (2007).

Festschrift honouring Professor David F. Williams, pp. 5068 -5073. DOI: 10 . 1016/j.biomaterials.2007.07.042.

9. R. A. Perez, J. Won, J. C. Knowles, and H. Kim. "Naturally and synthetic smart composite biomaterials for tissue regeneration." Advanced Drug Delivery Reviews, 65(4), (2013), pp. 471 -496. DOI: 10.1016/j.addr.2012.03.009.

10. J. Awrejcewicz, V. A. Krysko, S. P. Pavlov, M. V. Zhigalov, and A. V. Krysko. "Mathematical model of a three-layer micro- and nano-beams based on the hypotheses of the Grigolyuk-Chulkov and the modified couple stress theory." International Journal of Solids and Structures, 117, (2017), pp. 39 -50. DOI: 10.1016/j.ijsolstr.2017.04.011.

11. S. Bornassi and H. Haddadpour. "Nonlocal vibration and pull-in instability analysis of electrostatic carbon-nanotube based NEMS devices." Sensors and Actuators A: Physical, 266, (2017), pp. 185 -196. DOI: 10.1016/j.sna.2017.08.020. 
12. D. He, W. Yang, and W. Chen. "A size-dependent composite laminated skew plate model based on a new modified couple stress theory." Acta Mechanica Solida Sinica, 30(1), (2017), pp. 75 -86. DOI: 10.1016/j.camss.2016.12.001.

13. F. Villanueva-Flores, A. Castro-Lugo, O. T. Ramírez, and L. A. Palomares. "Understanding cellular interactions with nanomaterials: towards a rational design of medical nanodevices." Nanotechnology 31.13 (2020), p. 132002. DOI: 10.1088/ 1361-6528/ab5bc8

14. A. Farrokhabadi, A. Koochi, A. Kazemi, and M. Abadyan. "Effects of sizedependent elasticity on stability of nanotweezers." Applied Mathematics and Mechanics, 35(12), (2014), 1573 - 1590. DOI: 10.1007/s10483-014-1880-6.

15. F. Ebrahimi and R. E. Fardshad. "Modeling the size effect on vibration characteristics of functionally graded piezoelectric nanobeams based on Reddy's shear deformation beam theory." Advances in Nano Research, 6(2), (2018), pp. 113 -133. DOI: $10.12989 / \mathrm{anr}$.2018.6.2.113.

16. P. Dogra, J. D. Butner, Y. L. Chuang, S. Caserta, S. Goel, C. J. Brinker, V. Cristini, and Z. Wang. "Mathematical modeling in cancer nanomedicine: a review." Biomedical Microdevices, 21(2), (2019). DOI: 10.1007/s10544-0190380-2.

17. A. H. Faramarzi, T. Kaghazchi, H. A. Ebrahim, and A. A. Ebrahimi. "A mathematical model for prediction of pore size distribution development during activated carbon preparation." Chemical Engineering Communications, 202(2), (2015), pp. 131-143. DOI: 10.1080/00986445.2013.830609.

18. K. C. Wu, C. Y. Yang, and C. M. Cheng. "Using cell structures to develop functional nanomaterials and nanostructures-case studies of actin filaments and microtubules." Chemical Communications (Cambridge, England), 50(32), (2014), 4148 - 4157. DOI: 10.1039/c4cc00005f.

19. B. Karami, M. Janghorban, and A. Tounsi. "Variational approach for wave dispersion in anisotropic doubly-curved nanoshells based on a new nonlocal strain gradient higher order shell theory." Thin-Walled Structures, 129, (2018), pp. 251 -264. DOI: $10.1016 /$ j.tws.2018.02.025.

20. F. Kheibari and Y. T. Beni. "Size dependent electro-mechanical vibration of single-walled piezoelectric nanotubes using thin shell model." Materials and Design, 114, (2017), pp. 572 -583. DOI: https://doi.org/10.1016/j.matdes. 2016.10 .041 .

21. Y. T. Beni. "Size-dependent electromechanical bending, buckling, and free vibration analysis of functionally graded piezoelectric nanobeams." Journal of Intelligent Material Systems and Structures, 27(16), (2016), pp. 2199 -2215. DOI: 10.1177/1045389X15624798.

22. M. Esmaeili and Y. T. Beni. "Vibration and buckling analysis of functionally graded flexoelectric smart beam." Journal of Applied and Computational Mechanics, 5(5), (2019), pp. 900 -917. DOI: 10.22055/jacm.2019.27857.1439.

23. R. Hedayati, M. Sadighi, M. Mohammadi-Aghdam, and A.A. Zadpoor. "Analytical relationships for the mechanical properties of additively manufactured porous biomaterials based on octahedral unit cells." Applied Mathematical Modelling, 46, (2017), pp. 408 -422. DOI: 10.1016/j .apm.2017.01.076. 
24. H. Xe. "Modal decoupling using the method of weighted residuals for the nonlinear elastic dynamics of a clamped laminated composite." Mathematical Problems in Engineering, 46, (2009), pp. 1 -19. DOI: 10.1155/2009/972930.

25. J. E. Cicelia. "Solution of weighted residual problems by using Galerkin's method." Indian Journal of Science and Technology, 7(35), (Feb. 2014), pp. 52 -54. DOI: 10.13140/2.1.3364.2883

26. M. G. Sobamowo, Akanmu J. O., O. A. Adeleye, and A. A. Yinusa. "Nonlinear vibrations of single- and double-walled carbon nanotubes resting on two-parameter foundation in a magneto-thermal environment." SN Applied Sciences, 1, (2019). DOI: $10.1007 / \mathrm{s} 42452-019-1158-0$.

27. M. G. Sobamowo, O. M. Kamiyo, and O. A. Adeleye. "Further study on thermal performance of porous fin with temperature-dependent thermal conductivity and internal heat generation using Galerkin's method of weighted residual." Scientific News, WSN, 138(2), (2019), pp. $167-191$.

28. B. V. Reddy and K. R. Babu. "Application of variational methods and Galerkin method in solving engineering problems represented by ordinary differential equations." International Journal of Mechanical and Production Engineering, 4(4), (2016), pp. $75-80$.

29. Y. Wang and B. Guo. "Petrov-Galerkin methods for nonlinear systems without monotonicity." Applied Numerical Mathematics, 36(1), (2001), pp. 57 -78. DOI: https://doi.org/10.1016/S0168-9274(99)00054-9.

30. J. K. Zhou. Differential Transformation and its Applications for Electrical Circuits. Huazhong University Press, Wuhan, China, 1986.

31. M. M. Rashidi, O. Anwar Bég, and N. Rahimzadeh. "A generalized differential transform method for combined free and forced convection flow about inclined surfaces in porous media." Chemical Engineering Communications, 199,(2) (2012), pp. 257 -282. DOI: 10.1080/00986445.2011.586757.

32. Bor-Lih Kuo. "Application of the differential transformation method to the solutions of the free convection problem." Applied Mathematics and Computation, 165(1), (2005), pp. 63 -79. DOI: 10.1016/j .amc.2004.04.090.

33. M. Hatami and D. Jing. "Differential transformation method for Newtonian and non-Newtonian nanofluids flow analysis: Compared to numerical solution." Alexandria Engineering Journal, 55(2), (Jan. 2013), 731 - 739. DOI: 10.1016/ j.aej.2016.01.003.

34. O. A. Adeleye, O. Ipinnimo, A. Yinusa, and P. E. Otobo. "Differential transformation method for Newtonian and non-Newtonian nanofluids flow analysis: Compared to numerical solution." Journal of Applied Computational Mechanics, 6(4), (2020), 893 - 907. DOI: 10.22055/jacm.2019.29361.1589.

35. M. Cakir and D. Arslan. "The Adomian Decomposition Method and the Differential Transform Method for numerical nolution of multi-pantograph delay differential equations." Applied Mathematics, 06(8), (2015), pp. 1332-1343. DOI: $10.4236 / \mathrm{am} .2015 .68126$ 
36. S. Ghafoori, M. Motevalli, M. G. Nejad, F. Shakeri, D. D. Ganji, and M. Jalaal. "Efficiency of differential transformation method for nonlinear oscillation: Comparison with HPM and VIM." Current Applied Physics, 11(4), (2011), pp. 965 -971. DOI: $10.1016 /$ j.cap.2010.12.018.

37. S. Nourazar and A. Mirzabeigy. "Approximate solution for nonlinear Duffing oscillator with damping effect using the modified differential transform method." Scientia Iranica, 20(2), (2013), pp. 364 -368. DOI: 10.1016/j.scient.2013. 02.023

38. V. S. Erturk, Z. M. Odibat, and S. Momani. "The multi-step differential transform method and its application to determine the solutions of non-linear oscillators." Advances in Applied Mathematics and Mechanics, 4(4), (2012), pp. 422 -438. DOI: $10.1017 /$ S2070073300001727.

39. L. Kiss and G. Szeidl. "Vibrations of pinned-pinned heterogeneous circular beams subjected to a radial force at the crown point." Mechanics Based Design of Structures and Machines: An International Journal, 43(4), (2015), pp. 424449. DOI: $10.1080 / 15397734.2015 .1022659$.

40. G. Szeidl and L. Kiss. Theory of Vibrations, an Introduction. Series: Foundations of Engineering Mechanics, Series Editor: Vladimir I. Babitsky. Switzerland: Springer Nature, 2020. DOI: 10.1007/978-3-030-45074-8.

41. Sahmani S. and Aghdam M. M. "Size-Dependent Nonlinear Mechanics of Biological Nanoporous Microbeams." Nanomaterials for Advanced Biological Applications. Ed. by Rahmandoust M. and Ayatollahi M. Vol. 104. Advanced Structured Materials. Springer, 2019. DOI: 10.1007/978-3-030-10834-2_7. 